\title{
Indigenous Epidemiology: Identifying Health Disparities and Health Priorities
}

\section{Adrian Dominguez, Abigail Echo-Hawk, Kelsey Liu}

Urban Indian Health Institute, Seattle, Washington, United States

\section{Objective}

To create an interactive, user friendly platform for partners and data users to increase awareness around relevant health disparities and strengths within the urban AI/AN community.

\section{Introduction}

Historically, there has been a lack of data available to assess the health and well-being of urban American Indian/Alaska Natives (AI/ANs) in the United States. Furthermore, there has been limited data showing the increasing disparities that exists between urban AI/ANs and non-Hispanic Whites (NHW). Organizations looking to address these disparities have limited resources and mechanisms to address this rising problem [1]. In 2017, Urban Indian Health Institute (UHI) released a series of Community Health Profiles (CHPs) detailing the health status of urban AI/AN communities to provide detailed information to assist in program planning, grant writing and advocacy through increased documentation of current health disparities faced by urban AI/AN populations.

\section{Methods}

Data from 2010-2014 from several national data sources was analyzed using StataSE version 13 or SAS version 9.4. Using Tableau 10.1 this data was translated into an interactive dashboard to provide data on demographics, social determinants of health, mortality, sexually transmitted infections, maternal and child health, substance use, and mental health. These indicators were selected based off of Healthy People 2020 goals and examined disparities that exist between urban Native populations compared to NHW populations in the same areas. By creating an online data tool, the dashboard is able to provide information and data regarding descriptive statistics on social and health disparities, and can be used to assist public health professionals, community members, and $\mathrm{AI} / \mathrm{AN}$ organizations with program planning and interventions.

\section{Results}

Key findings from the results showed that urban AI/AN people continue to face significant socio-economic disparities when compared to non-Hispanic whites, for example more than twice as many urban AI/ANs over 16 were unemployed between 20102014 when compared to their NHW counterparts (15.8\% vs. 7.4\%). However, urban AI/ANs experience significantly lower rates of suicide when compared to NHW communities. Additionally, although urban AI/AN women gave birth at younger ages on average when compared to NHW women of reproductive age, they were significantly less likely to deliver by cesarean section.

Lastly, significantly fewer urban AI/ANs reported using alcohol in the past month when compared to NHWs (44\% vs. 60\%).

\section{Conclusions}

This analysis of national surveillance data highlights the strengths of the urban AI/AN community around suicide and alcohol use in the past month and point to areas for improvement. By translating these results into a Tableau dashboard, this data is more user friendly and can be used to support programs in identifying health priorities.

\section{Acknowledgement}

This project was supported by the Indian Health Service via funding through grants (entitled "Epidemiology Program for American Indian/ Alaska Native Tribes and Urban Indian Communities"). 


\section{References}

1. NCAI Policy Research Center. Retrieved July 23, 2018, from http://www.ncai.org/policy-researchcenter/research-data/data 